\title{
HAUSDORFF TRANSFORMS OF BOUNDED SEQUENCES
}

\author{
J. H. WELLS
}

Let $\phi$ be a complex valued function of bounded variation on $[0,1]$ such that $\phi(0)=0$. The statement that $H(\phi)$ is the Hausdorff transformation generated by $\phi$ means that if $x$ is a complex number sequence, then $y=H(\phi) x$ where

$$
v_{n}=\int_{0}^{1} \sum_{p=0}^{n} C_{n, p} p^{p}(1-t)^{n-p} x_{p} d \phi(t) \quad(n=0,1,2, \cdots) .
$$

The sequence is said to be $H(\phi)$ evaluable to $L$ if $y_{n} \rightarrow L$ as $n \rightarrow \infty$. The transformation $H(\phi)$ is regular (i.e., $\lim x_{n}=L$ implies $\lim y_{n}=L$ ) if and only if $\phi(0+)=0$ and $\phi(1)=1$. If $x$ is a complex number sequence, then $C(x)$ denotes the set of limit points of $x$.

Among the regular Hausdorff transformations are the methods $C_{r}(\operatorname{Re} r>0)$ of Cesàro, $H_{r}(\operatorname{Re} r>0)$ of Hölder and $E_{r}(0<r<1)$ of Euler for which $\phi(t)=1-(1-t)^{r}$,

$$
\phi(t)=\frac{1}{\Gamma(r)} \int_{0}^{t}(\log 1 / u)^{r-1} d u, \text { and } \phi(t)= \begin{cases}0, & 0 \leqq t<r \\ 1, & r \leqq t<1\end{cases}
$$

respectively. It has been shown by Barone [2] that the regular Hausdorff transformations $C_{r}(\operatorname{Re} r>0), H_{r}(r$ a positive integer) and $E_{r}(0<r<1)$ have the property that the set of limit points of the transform of each bounded complex number sequence is connected. In each case the result was obtained by use of

THEOREM A. If $y$ is a bounded complex number sequence such that $\left|y_{n}-y_{n-1}\right| \rightarrow 0$ as $n \rightarrow \infty$, then $C(y)$ is connected.

The object of this note is to prove the following

THEOREM. If $H(\phi)$ is a regular Hausdorff transformation, then the following two statements are equivalent:

(i) $\phi\left(1^{-}\right)=\phi(1)$,

(ii) $C(H(\phi) x)$ is connected for each bounded complex number sequence $x$.

1. Proof that (i) implies (ii). If $x$ is a bounded complex number sequence, then $y=H(\phi) x$ is bounded and for each positive integer $n$

Presented to the Society, April 25, 1959; received by the editors March 30, 1959. 
$\left|y_{n}-y_{n-1}\right|$

$$
\leqq M \int_{0}^{1} \sum_{p=0}^{n}\left|C_{n, p} t^{p}(1-t)^{n-p}-C_{n-1, p} t^{p}(1-t)^{n-1-p}\right||d \phi(t)|
$$

where $M=1$.u.b. $\left|x_{p}\right|$ and $C_{n-1, n} t^{n}(1-t)^{-1}=0(0 \leqq t \leqq 1)$. If $b_{n}(t)$ denotes the largest integer which does not exceed $n t$, we have $[2$, p. 751$]$

$$
\left|y_{n}-y_{n-1}\right| \leqq 2 M \int_{0}^{1} C_{n-1, b_{n}(t)} t^{b_{n}(t)+1}(1-t)^{n-1-b_{n}(t)}|d \phi(t)| \text {. }
$$

Let $\epsilon>0$. Since $\phi\left(0^{+}\right)=\phi(0)$ and $\phi\left(1^{-}\right)=\phi(1)$ we can choose $\delta>0$ so that

$$
2 M \int_{0}^{\delta}|d \phi(t)|<\epsilon / 3 \text { and } 2 M \int_{1-\delta}^{1}|d \phi(t)|<\epsilon / 3
$$

An application of Stirling's formula shows that there exists a positive number $k$ such that, if $1<n \delta$,

$$
C_{n-1, b_{n}(t)} t^{b_{n}(t)+1}(1-t)^{n-1-b_{n}(t)}<k \frac{(n-1)^{n-1 / 2}}{(n-1 / \delta)^{n}} \quad(\delta \leqq t \leqq 1-\delta) .
$$

Consequently, if $v$ denotes the total variation of $\phi$ on $[0,1]$,

$$
\left|y_{n}-y_{n-1}\right| \leqq \epsilon / 3+2 M v k \frac{(n-1)^{n-1 / 2}}{(n-1 / \delta)^{n}}+\epsilon / 3<\epsilon
$$

for $n$ sufficiently large. Thus $\left|y_{n}-y_{n-1}\right| \rightarrow 0$ as $n \rightarrow \infty$ so that, by Theorem A, $C(y)$ is connected. Hence (i) implies (ii).

2. Proof that (ii) implies (i). Suppose there exists a regular Hausdorff transformation $H(\phi)$ with $\phi\left(1^{-}\right) \neq \phi(1)$ such that $C(H(\phi) x)$ is connected for each bounded complex number sequence $x$. It is sufficient to assume $\phi$ real valued. Let $\phi=\phi_{1}+\phi_{2}$ where

$$
\phi_{1}(t)=\left\{\begin{array}{ll}
\phi(t), & 0 \leqq t<1, \\
\phi\left(1^{-}\right) & t=1,
\end{array} \quad \phi_{2}(t)=\left\{\begin{array}{l}
0, \quad 0 \leqq t<1, \\
\phi(1)-\phi\left(1^{-}\right), \quad t=1
\end{array}\right.\right.
$$

and for each pair $n, p$ of nonnegative integers, $p \leqq n$, let

$$
A_{n p}=\int_{0}^{1} C_{n, p} t^{p}(1-t)^{n-p} d \phi_{1}(t) .
$$

It is not difficult to show [3, pp. 308-309] that (1) $A_{n p} \rightarrow 0$ as $n \rightarrow \infty$ $(p=0,1,2, \cdots)$. If $n$ is a nonnegative integer and $\epsilon>0$, then 


$$
\begin{aligned}
\left|A_{n+p, p}\right| & \leqq(n+p)^{n} \int_{0}^{\delta} t^{p}\left|d \phi_{1}(t)\right|+\int_{\delta}^{1}\left|d \phi_{1}(t)\right| \\
& \leqq(n+p)^{n} \delta^{p} \int_{0}^{\delta}\left|d \phi_{1}(t)\right|+\int_{\delta}^{1}\left|d \phi_{1}(t)\right|<\epsilon
\end{aligned}
$$

for $p$ sufficiently large, provided $\delta$ is a number in $(0,1)$ so chosen that

$$
\int_{\delta}^{1}\left|d \phi_{1}(t)\right|<\epsilon / 2 \text {. }
$$

Hence (2) $A_{n+p, p} \rightarrow 0$ as $p \rightarrow \infty(n=0,1,2, \cdots)$. Suppose each of $n$ and $p$ is a positive integer. Since the function $C_{n+p, p} t^{p}(1-t)^{n}$ is monotone on each of the intervals $[0, p /(n+p)]$ and $[p /(n+p), 1]$ we have, after an integration by parts, the inequality

$$
\begin{aligned}
\left|A_{n+p, p}\right| \leqq & \int_{0}^{p /(n+p)}\left|\phi_{1}(t)\right| d\left[C_{n+p, p} t^{p}(1-t)^{n}\right] \\
& +\int_{p /(n+p)}^{1}\left|\phi_{1}(t)\right| d\left[-C_{n+p, p} t^{p}(1-t)^{n}\right] \\
\leqq & 2 M C_{n+p, p}[p /(n+p)]^{p}[n /(n+p)]^{n}<k(1 / n+1 / p)^{1 / 2}
\end{aligned}
$$

where $M=1$.u.b.0 $\leq \leq 1\left|\phi_{1}(t)\right|$ and $k$ is a constant arising from an application of Stirling's formula.

From conditions (1), (2) and (3) it follows that $A_{n p} \rightarrow 0$ as $n \rightarrow \infty$ uniformly with respect to $p$. According to a result of Agnew [1] this property of the numbers $A_{n p}$ is sufficient to ensure the existence of a divergent sequence $x$ of zeros and ones such that $H\left(\phi_{1}\right) x$ has limit zero. Therefore $y=H(\phi) x=H\left(\phi_{1}\right) x+H\left(\phi_{2}\right) x$ is the sum of a sequence with limit zero and a divergent sequence each element of which is zero or $\phi_{2}(1)$. Consequently, $C(y)$ is not connected. This is a contradiction. Hence (ii) implies (i).

\section{REFERENCES}

1. R. P. Agnew, $A$ simple sufficient condition that a method of summability be stronger than convergence, Bull. Amer. Math. Soc. vol. 52 (1946) pp. 128-132.

2. H. G. Barone, Limit points of sequences and their transforms by methods of summability, Duke Math. J. vol. 5 (1939) pp. 740-752.

3. H. S. Wall, Analytic theory of continued fractions, New York, 1948.

The University of North Carolina 